\title{
TRACOMA: uma antiga patologia ainda negligenciada na atualidade.
}

\author{
Evanildo José da SILVA ${ }^{1}$ \\ Layze Alves Vieira OLIVEIRA ${ }^{2}$ \\ Daisy de Rezende Figueiredo FERNANDES ${ }^{3}$ \\ Cleya da Silva Santana CRUZ \\ Leida Calegário de OLIVEIRA ${ }^{5}$
}

\begin{abstract}
${ }^{1}$ Médico, Mestre pelo Programa de Pós-Graduação em Saúde, Sociedade e Ambiente (PPGSaSA) e Docente da Faculdade de Medicina de Diamantina. Universidade Federal dos Vales do Jequitinhonha e Mucuri (UFVJM). evanildo.silva@ufvjm.edu.br

${ }^{2}$ Acadêmica de Enfermagem - Faculdade de Ciências Biológicas e da Saúde(FCBS)/ UFVJM. layze.ufvjm@gsmail.com ${ }^{3}$ Enfermeira, Doutora . Docente do Departamento de Enfermagem - FCBS/UFVJM. daisygouveia@oi.com.br

${ }^{4}$ Enfermeira, Mestra pelo PPGSaSA/UFVJM. Superintendência Regional de Saúde de Diamantina da Secretaria de Estado de Saúde de Minas Gerais. joaquimcesar@yahoo.com.br

${ }^{5}$ Bióloga, Doutora. Docente do Departamento de Farmácia - FCBS/UFVJM. leida@ufvjm.edu.br
\end{abstract}

Recebido em: 15/10/2014 - Aprovado em: 15/06/2015 - Disponibilizado em: 15/07/2015

\section{RESUMO}

Considerado como a principal causa infecciosa de cegueira no mundo, o tracoma tem como agente etiológico a bactéria Chlamydia trachomatis e manifesta-se como uma inflamação crônica, gerando alterações cicatriciais que podem culminar com a cegueira. Esta doença está intimamente relacionada às más condições socioeconômicas e de saneamento da população. Embora relatos indiquem que seja uma doença conhecida há milênios, esta pode ser considerada como mundialmente negligenciada, o que é evidenciado pelo baixo investimento em pesquisas que visem melhorias tanto do diagnóstico quanto do tratamento. Realizou-se um levantamento no dia 10/05/2014 nas bases de referências bibliográficas: Medical Literature Analysis and Retrieval System Online - MEDLINE (PUBMED), Literatura Latino-Americana e do Caribe em Ciências da Saúde (LILACS) e Scientific Electronic Library Online (Scielo), onde foram pesquisados trabalhos publicados no período de janeiro/2004 a dezembro/2013. Digitaram-se separadamente as palavras: Tracoma, Glaucoma e Catarata e em seguida fez-se as análises dos dados obtidos. Foram considerados todos os trabalhos publicados no período.

Palavras -Chave: Tracoma. Glaucoma. Catarata. Negligência. Cegueira.

\begin{abstract}
Considered the leading infectious cause of blindness in the world, trachoma is the etiologic agent the bacterium Chlamydia trachomatis and it is manifested as a chronic inflammation, causing scarring changes that may lead to blindness. This disease is closely related to poor socioeconomic conditions and sanitation of the population. Although reports indicate that it is a disease known for millennia, it can be considered as globally neglected, as evidenced by the low investment in research aimed at improvements in both the diagnosis and the treatment. We conducted a survey on 05.10.2014 in the references bases : Medical Literature Analysis and Retrieval System Online - MEDLINE ( PubMed ), Latin American and Caribbean Health Sciences ( LILACS) and Scientific Electronic Library Online ( SciELO ), which were surveyed studies published from January/2004 to December/2013 .The words were typed separately : Trachoma , Glaucoma and Cataract and then became the analysis of the data. We considered all studies published in the period.
\end{abstract}

Keywords: Trachoma . Glaucoma. Cataract . Negligence. Blindness . 


\section{INTRODUÇÃO}

Entre os órgãos sensoriais, a visão é considerada como o meio mais importante de interação do ser humano com o ambiente. $\mathrm{O}$ sistema visual inicia-se no globo ocular e estende-se até o córtex occipital, na chamada via óptica.

O olho humano encontra-se no interior da órbita e, em sua parte anterior, é recoberto externamente pelas pálpebras superior e inferior, onde tem-se o tarso que funciona como esqueleto.

O globo ocular (Fig.1A) é formado basicamente por três camadas ou túnicas, que são:

- Esclerótica: mais externa, na parte anterior é transparente e denomina-se córnea e na posterior, que é opaca, esclera. O limite entre ambas é uma circunferência chamada de limbo;

- Úvea: camada média, na parte posterior é denominada coróide e, na anterior íris, onde se tem no centro a pupila;

- Retina: mais interna, é a camada neuro-sensorial, onde se encontram células chamadas cones e bastonetes, que são responsáveis pela percepção da visão.

A face interna das pálpebras e a esclera são recobertas por uma membrana fina e transparente chamada conjuntiva (Fig.1B), onde encontramos muitos dos sinais clínicos que evidenciam diversas patologias oculares (GUYTON; HALL， 2011; RIORDAN-EVA; WHITCHER， 2011; YANOFF; DUKER, 2011; PUTZ et al., 2011).

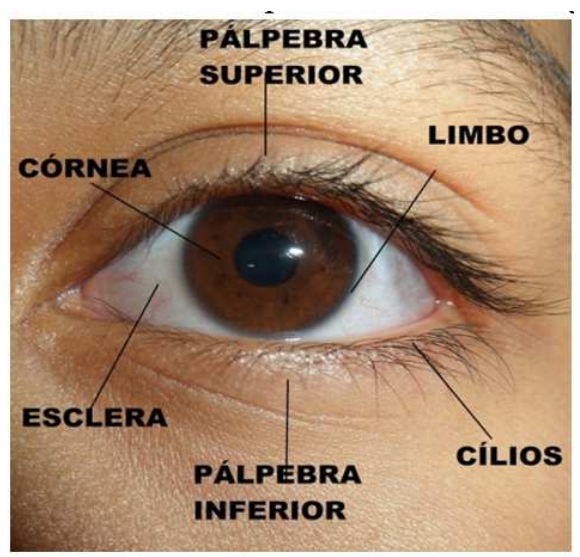

A- Anatomia do Olho

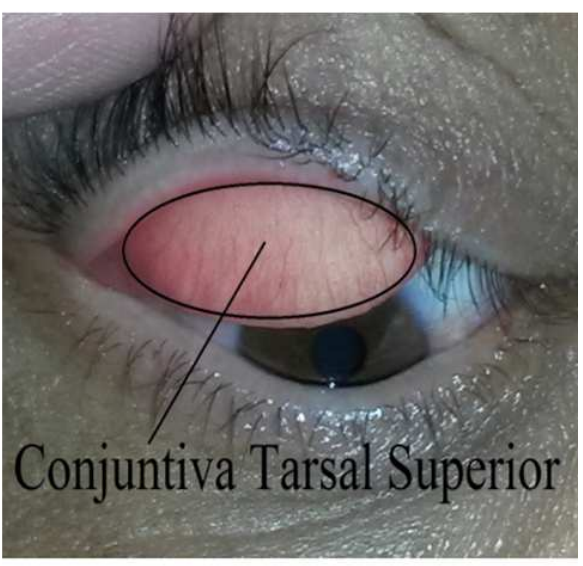

B -ConjuntivaTarsal Superior

FIGURA 1 - Detalhes do olho humano. Fonte: Arquivo Pessoal do Autor.

Nas últimas décadas tem sido observado um considerável desenvolvimento no diagnóstico e tratamento de grande parte das doenças oculares, na maioria das vezes atrelado aos interesses comerciais das grandes indústrias farmacêuticas e de equipamentos. Entretanto, no que se refere às doenças negligenciadas, verifica-se um baixo investimento, pois estas atingem camadas 
mais carentes da população, gerando, assim, menor possibilidade de retorno econômico.

\section{O Tracoma}

\section{Histórico}

O termo Tracoma origina-se do grego Trachomas, cujo significado é rugoso, áspero, devido ao aspecto apresentado pela conjuntiva tarsal durante o curso desta doença.

É conhecido desde o início da humanidade, podendo-se encontrar relatos em várias civilizações, tais como China (sec XXVII a.C), Suméria (sec XXI a.C), Egito ( $\sec$ XIX a.C), Grécia (sec V a.C) e Roma (sec. I a.C). Disseminou-se pelo antigo continente europeu com as grandes migrações dos povos na segunda metade do século XIX e início do XX (BRASIL, 2001).

Essa doença teve alta prevalência no mundo islâmico e na Grécia durante a idade média, quando foi levada para a Europa e África, tornando-se endêmica. Neste mesmo período, a partir da Europa, devido à colonização, foi trazido para o continente americano. Na segunda metade do século XIX e início do XX, achava-se amplamente disseminado pelo mundo, então, durante o século XX, com a melhoria das condições de vida, desapareceu da Europa, América do Norte e Japão (BRASIL, 2001). Entretanto, persiste como um importante problema de saúde pública nos países em desenvolvimento (LAVETT et al, 2013; WORLD HEALTH ORGANIZATION, 2012; MARIOTTI;
PASCOLINI; ROSE-NUSSBAUMER, 2009; POLACK et al., 2005).

Acredita-se que o Tracoma tenha sido trazido ao nordeste do Brasil no século XVIII por ciganos expulsos de Portugal, estabelecendo-se nos estados do Maranhão e Ceará. Outros focos importantes no país teriam ocorrido nos estados de São Paulo e Rio Grande do Sul, decorrentes da imigração européia no século XIX (BRASIL, 2001).

Em 1943, o Governo Federal organizou a campanha nacional contra $\mathrm{O}$ tracoma (BRASIL, 2001), mas apesar de várias tentativas de contenção, a doença se espalhou pelo Brasil.

Durante o período que ficou conhecido como "milagre econômico brasileiro (décadas de 50 a 70), foi observada uma diminuição na detecção da doença no país, fazendo acreditar que o Tracoma não mais constituía problema de saúde pública, o que Schellini e Sousa (2012) chamaram de "mito da erradicação do Tracoma" pelo fato das pesquisas epidemiológicas demonstrarem que o tracoma persistiu em nosso país (JESUS et al., 2013; LOPES et al., 2013; DANTAS, 2013; MACHARELLI et al., 2013; CANINÉO et al., 2012; SCHELLINI et al., 2010; FERRAZ et al., 2010; LUCENA; CRUZ; CAVALCANTI et al., 2010 ; LOPES, 2008; ALMEIDA, 2007; KOIZUMI et al., 2005).

$\mathrm{O}$ equívoco quanto à erradicação ainda permanece na comunidade científica, o que levou à negligência no enfrentamento à 
doença, bem como no ensino da sua etiologia, diagnóstico e tratamento nos cursos de medicina e especialização em oftalmologia no país (SCHELINNI; SOUSA, 2012)

Considerado como a principal causa infecciosa de cegueira no mundo, o tracoma tem como agente etiológico a bactéria Chlamydia trachomatis e manifesta-se como uma inflamação crônica e recidivante da conjuntiva e córnea, gerando alterações cicatriciais que podem levar à formação de entrópio (pálpebra invertida), triquíase (cílios invertidos) e opacidade de córnea, podendo culminar com a cegueira (SCHELLINI, 2012; GUYTON; HALL, 2011; RIORDAN-EVA; WHITCHER, 2011; YANOFF; DUKER, 2011). Embora relatos indiquem que seja uma doença conhecida há milênios (BRASIL, 2001), esta pode ser considerada como mundialmente negligenciada.

\section{Distribuição Geográfica}

O Tracoma é considerado endêmico em mais de 50 países. Na figura 2 vemos a distribuição geográfica do Tracoma no mundo.

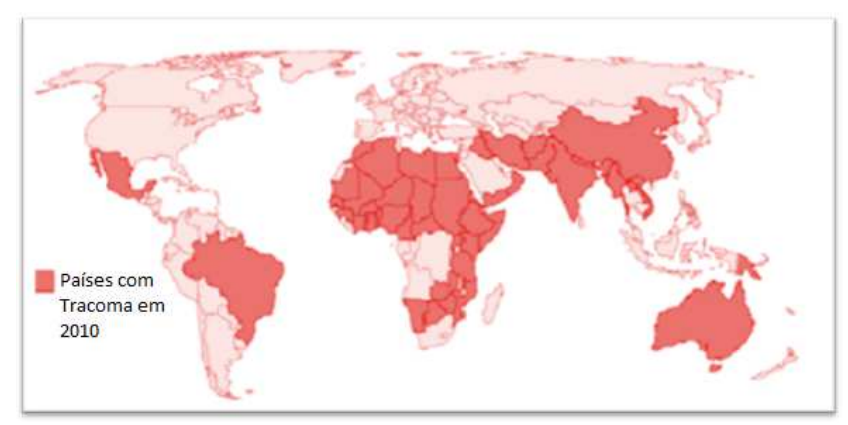

FIGURA 2 - Mapa de distribuição da ocorrência de Tracoma ativo no mundo, 2010.

Fonte: World Health Organization, 2012.
Conforme pode ser observado na figura 2, em 2010 o tracoma encontrava-se presente principalmente na Ásia, África, Oriente Médio e Índia, existindo ainda em menor proporção na América Latina e Oceania (World Health Organization, 2012). Em outras regiões do globo, devido à falta de relatos ou de diagnóstico, não foram descritos casos da doença. Pesquisas epidemiológicas comprovam a existência do Tracoma na realidade de todos os estados brasileiros nos quais foi feita investigação da doença, com taxas de detecção variáveis (JESUS et al., 2013; LOPES et al., 2013; MACHARELLI et al., 2013; CANINÉO et al., 2012; SCHELLINI et al., 2010; LUCENA; CRUZ; CAVALCANTI et al., 2010; FERRAZ et al., 2010; DAMASCENO et al., 2009; LOPES, 2008; ALMEIDA, 2007; KOIZUMI, 2005; PAULA; MEDINA; CRUZ, 2002).

\section{Agente Etiológico, Transmissão e Patogenia}

O agente etiológico do Tracoma é a bactéria Chlamydia trachomatis que é considerada parasita intracelular obrigatório, possuindo corpúsculos de inclusão citoplasmáticos. Classificada como bactéria gram-negativa, esta apresenta 19 sorotipos diferentes já identificados. Os sorotipos de $C$. trachomatis são agrupados em três sorogrupos: sorogrupo B (sorotipos B, Ba, D, Da, E, L1, L2 e L2a), sorogrupo intermediário (F e G) e sorogrupo C (sorotipos A, C, H, I, Ia, J, Ja, K e L3) (MILLMAN; TAVARE et 
al., 2001). Os sorotipos A, B, Ba e C causam o tracoma, os sorotipos L1, L2, L2a e L3 causam o linfogranuloma venéreo e os de $\mathrm{D}$ a $\mathrm{K}$ (incluindo $\mathrm{Da}$, Ja e Ia) causam outras doenças sexualmente transmissíveis (MOLINA; GUERRA; LLORENTE，2013; PUTZ, 2011; DEAN et al., 2009).

O Tracoma é transmitido, principalmente, de forma direta de indivíduo para indivíduo, através do contato com secreções oculares e, secundariamente, de forma indireta, através de objetos contaminados (toalhas, lençóis e fronhas). A mosca doméstica (Musca domestica) e, ou a lambe-olhos (Hippelates sp.) podem também atuar como vetores da doença (REILLY et al., 2007).

A doença inicia-se sob a forma de uma conjuntivite folicular, cuja característica principal é a presença de folículos, pequenos nódulos esbranquiçados circundados por vasos sanguíneos, e de infiltrado inflamatório difuso, que se estende por toda a conjuntiva, especialmente na palpebral superior. Nos casos mais brandos, os folículos podem regredir espontaneamente. Nos casos mais severos, eles crescem, evoluindo para necrose com formação de pequenos pontos cicatriciais na conjuntiva. Depois de repetidas reinfecções, forma-se um número cada vez maior de pontos cicatriciais, levando à formação de cicatrizes mais extensas. Essas cicatrizes podem tracionar a pálpebra superior, levando à sua distorção e causando o entrópio (inversão na posição palpebral), fazendo com que os cílios invertidos toquem no globo ocular. A triquíase caracteriza-se pelo nascimento de cílios anômalos que crescem invertidos, voltando-se para a córnea. Essas alterações podem provocar ulcerações corneanas, com consequente opacificação, que pode levar a graus variados de diminuição da acuidade visual e cegueira (THYLEFORS et al., 1987).

\section{Sinais, Sintomas e Apresentações Clínicas} do Tracoma

A sintomatologia associada ao tracoma inflamatório é inespecífica, podendo haver lacrimejamento, sensação de corpo estranho, fotofobia discreta e prurido. Um grande número de casos de tracoma, principalmente entre as crianças mais jovens, é assintomático. Os doentes que apresentam entrópio e, ou triquíase e aqueles com ulcerações corneanas, referem dor constante e intensa fotofobia. Infecções bacterianas secundárias podem estar associadas ao quadro, contribuindo para a disseminação da doença (GUYTON; HALL, 2011; RIORDAN-EVA; WHITCHER， 2011; YANOFF; DUKER, 2011; PUTZ et al., 2011; BRASIL, 2001).

A identificação precoce dos pacientes acometidos é importante evitando que estes evoluam para alterações mais graves.

De acordo com Thylefors et al. (1987) o tracoma apresenta cinco formas clássicas (Fig.3). Duas delas representam a fase 
inflamatória, quando pode haver transmissão da doença e as outras três são sequelares, quando não há transmissão.
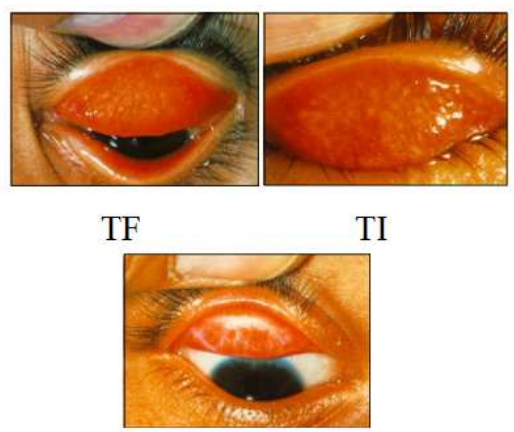

TS

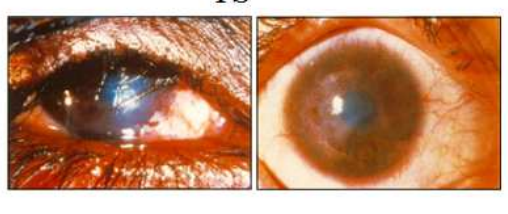

TT

OP

FIGURA 3 - Formas Clínicas de Apresentação do Tracoma (TF: Tracoma Inflamatório Folicular; TI: Tracoma Inflamatório Intenso; TS: Tracoma Cicatricial Conjuntival; TT: Triquíase Tracomatosa; OP: Opacificação corneana).

Fonte:Brasil, 2001

Os dois tipos de formas inflamatórias são: - Tracoma Inflamatório Folicular (TF): com moderado grau de infiltração difusa, deve-se observar a presença de pelo menos 5 folículos de no mínimo $0,5 \mathrm{~mm}$ de diâmetro na conjuntiva tarsal superior. Os folículos são arredondados, mais pálidos em relação à conjuntiva circundante e apresentam vaso sanguíneo na periferia ao seu redor;

- Tracoma Inflamatório Intenso (TI): há espessamento predominantemente difuso da Conjuntiva tarsal superior, que se apresenta geralmente enrugada e avermelhada, não permitindo a visualização de mais de $50 \%$ dos vasos tarsais profundos.

As três formas sequelares são:
- Tracoma Cicatricial Conjuntival (TS): as cicatrizes são facilmente visualizadas como linhas esbranquiçadas, fibrosas com bordas retas, angulares ou estreladas, dispostas de maneira vertical e horizontal;

- Triquíase Tracomatosa (TT): tem-se quando pelo menos um dos cílios atrita o globo ocular, ou há evidência de recente remoção de cílios invertidos;

- Opacificação Corneana (CO): facilmente visualizada, deve apresentar um leucoma com intensidade suficiente para obscurecer ao menos uma parte da borda pupilar.

\section{Diagnóstico e Tratamento}

O diagnóstico do tracoma é essencialmente clínico e, geralmente, realizado por meio de exame ocular externo, utilizando lupa binocular de 2,0 a 3,0 vezes de aumento (THYLEFORS et al., 1987). A OMS orienta que o diagnóstico de Tracoma deve ser dado quando houver pelo menos dois dos seguintes sinais clínicos:

- folículos na conjuntiva tarsal superior;

- folículos no limbo (também chamados fossetas de Herbert);

- cicatriz conjuntival típica(pode ser vertical e,ou horizontal);

- pannus no limbo superior(invasão de vasos sanguíneos neo-formados).

Na década de 90, sob a liderança da OMS, foi criada a "Aliança Global para a Eliminação do Tracoma como causa de Cegueira até 2020", da qual o Brasil participa.

A OMS preconiza a estratégia $S A F E$, que 
significa: S (surgery: cirurgia, quando necessário), A (Antibiótic: uso de antibiótico), $\mathrm{F}$ (Face: limpeza facial) e $\mathrm{E}$ (enviroment: cuidados ambientais) (World Health Organization, 1997).

Atualmente, pela eficácia e praticidade, o tratamento de escolha é feito com o antibiótico Azitromicina $(20 \mathrm{mg} / \mathrm{Kg})$, em dose única, administrada por via oral em pó solúvel para crianças até 12anos e, ou 45 $\mathrm{Kg}$ de peso corporal ou através de comprimidos de 500mg para adultos e crianças maiores de 12 anos ou acima de 45 $\mathrm{kg}$ de peso corporal, conforme está contido na portaria $n^{\circ} 67$ de 22/12/2005 do Ministério da Saúde do Brasil (BRASIL, 2005).

Várias pesquisas com a finalidade de se produzir uma vacina eficiente contra Chlamydia têm sido realizadas e atualmente encontram-se em estágio avançado (HAFNER; WILSON; TIMMS, 2013). Certamente alcançar este objetivo seria de grande importância no combate a esta bactéria.

\section{OBJETIVOS}

Analisar o número de publicações científicas relativas ao tema Tracoma, comparativamente com outras doenças oculares, no período de janeiro/2004 a dezembro/2013.

\section{METODOLOGIA}

Trata-se de um estudo descritivo e comparativo onde foi realizado levantamento no dia 10/05/2014 nas bases de referências bibliográficas: Medical Literature Analysis and Retrieval System Online - MEDLINE (PUBMED), Literatura Latino-Americana e do Caribe em Ciências da Saúde (LILACS) e Scientific Electronic Library Online (Scielo), onde foram pesquisados trabalhos de qualquer natureza publicados entre janeiro/2004 a dezembro/2013. Digitaram-se separadamente as palavras: Tracoma, Glaucoma e Catarata. Foram considerados todos os trabalhos publicados no período.

\section{RESULTADOS E DISCUSSÃO}

A visão, citada inúmeras vezes ao longo da história e em verso e prosa por grandes artistas da humanidade, possui papel fundamental em nossas vidas, tanto nas interações com o meio ambiente, quanto nas relações interpessoais. Entretanto, pode ser prejudicada em virtude de diversas patologias.

Entre as doenças oculares tem-se: ametropias, catarata, glaucoma, pterígio, conjuntivites e uveíte, dentre as conjuntivites, encontra-se o Tracoma, doença negligenciada que cursa com surtos recidivantes de inflamação dos olhos.

Embora bastante prevalente em nosso meio (Silva, 2014; Jesus et al., 2013; Lopes et al., 2013; Lucena; Cruz; Cavalcanti, 2010; Lopes, 2008; Paula; Medina; Cruz; 2002), o Tracoma permanece como uma doença negligenciada. Por outro lado, doenças como glaucoma e catarata apresentam-se também 
bastante prevalentes (Machado et al, 2012; Couto Jr. et al., 2013), mas despertam maior interesse da indústria farmacêutica por gerar maior retorno financeiro.

A figura 4 apresenta os resultados da busca por trabalhos científicos publicados no período de janeiro/2004 a dezembro/2013 e que tratassem dos temas tracoma, glaucoma e catarata.

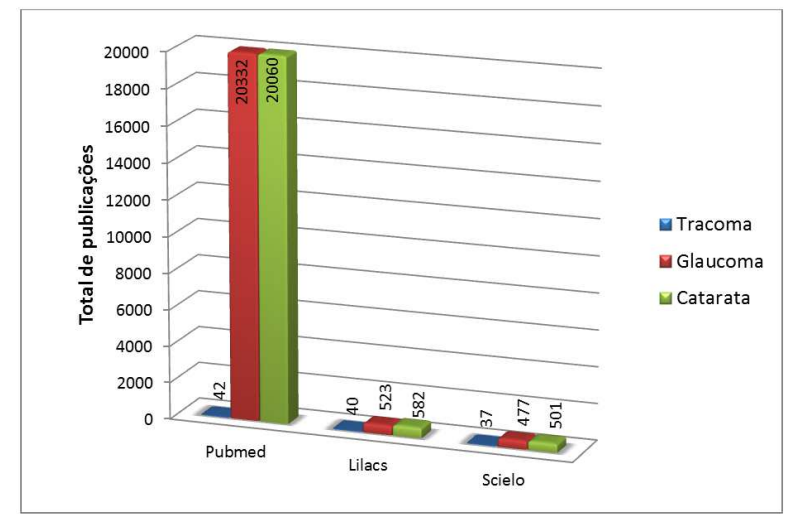

Figura 4- Levantamento do número de publicações sobre os temas tracoma, glaucoma e catarata, nos portais PUBMED, LILACS e SCIELO, no período de janeiro/2004 a dezembro/2013.

Fonte: Dados da Pesquisa

A análise da figura permite-nos observar grande discrepância no número de publicações quando se compara os temas Glaucoma e Catarata em relação a Tracoma. Observa-se um número relativamente baixo de publicações a respeito do Tracoma, o que agrava o quadro de desconhecimento pelos profissionais de saúde e população a respeito da doença. A tabela 1 apresenta a relação percentual entre o número de publicações relativas a estes temas. Pode-se perceber que na base de dados Pubmed as publicações relativas ao tema Tracoma foram apenas $0,21 \%$ daquelas relativas aos temas Glaucoma e Catarata. Nas bases Lilacs e Scielo o número de publicações sobre Tracoma foi, em média 7,42\% (dp 0,40\%) em relação a estas doenças.

Tabela 1. Percentual do número de publicações sobre o tema Tracoma em relação aos temas Glaucoma e Catarata nas bases de dados Pubmed, Lilacs e Scielo, relativas ao período de janeiro/2004 a dezembro/2013.

\begin{tabular}{lccc}
\hline $\begin{array}{l}\text { Relação } \mathbf{n}^{\mathbf{0}} \\
\text { publicações (\%) }\end{array}$ & Pubmed & Lilacs & Scielo \\
\hline Tracoma/Glaucoma & 0,21 & 7,65 & 7,76 \\
Tracoma/Catarata & 0,21 & 6,87 & 7,39 \\
\hline \multicolumn{2}{l}{ Fonte: Dados da Pesquisa }
\end{tabular}

Este fato evidencia a pouca importância dada ao Tracoma, uma vez que, por se tratar de doença que não gera grande lucro para a indústria farmacêutica e de equipamentos, não há interesse no patrocínio de novas pesquisas. O contrário ocorre com os temas glaucoma e catarata, uma vez que estes geram grande retorno econômico para as indústrias. Este dado sugere que há uma negligência não só das indústrias farmacêuticas e de equipamentos, mas também da academia em relação ao tema. Não existem na literatura estudos com levantamentos semelhantes a este que possam fornecer dados para análise comparativa.

\section{CONCLUSÕES}

Com o exposto acima, chama-se atenção para o problema da negligência, inclusive pela academia, em relação ao Tracoma, uma doença tida equivocadamente como extinta em nosso meio. O tema precisa 
ser abordado durante a formação dos profissionais de saúde, como forma de conscientização e disseminação do conhecimento, sendo que a academia tem papel primordial nesta missão. É necessário também o desenvolvimento de ações educativas em saúde, pois estas têm importante impacto nas atividades de prevenção e controle das doenças, mobilizando a comunidade, para criar recursos e participar ativamente do processo.

\section{REFERÊNCIAS BIBLIOGRÁFICAS}

ALMEIDA, F. L. B. Ocorrência de Complicações do Tracoma em Zona Endêmica de Baturité/Ceará. Dissertação (Mestrado) - Curso de Mestrado Acadêmico em Saúde Pública do Centro de Ciências da Saúde, Universidade Estadual do Ceará, Fortaleza, 2007.85p

BRASIL. Gabinete do Ministério. Ministério da Saúde. Fundação Nacional de Saúde. Manual de Controle do Tracoma. Brasília, 2001. 54p.

BRASIL. Gabinete do ministério. Portaria $\mathrm{n}^{\mathrm{o}}$ 67, de 22 de dezembro de 2005. Dispõe sobrea inclusão da Azitromicina no tratamento sistêmico de tracoma. Diário Oficial da União [da] República Federativa do Brasil. Brasília, $26 \mathrm{dez}$. 2005.

CANINÉO, P. A. et al. Inquérito epidemiológico de tracoma em escolares no município de Embu das Artes - SP. Arquivo Brasileiro de Oftalmologia. São Paulo, v. 75, n. 1, p. 264-266, 2012.

COUTO JR., A. S. et al . Prevalência de ametropias e oftalmopatias no quilombo São José da Serra - Valença - RJ. Revista brasileira de oftalmologia, Rio de Janeiro, v. 72, n. 6, Dec. 2013.
DAMASCENO, R. W. F. et al. Tracoma: estudo epidemiológico de escolares em Alagoas - Brasil. Arquivo Brasileiro de Oftalmologia, São Paulo, v. 72, n. 3, May./June 2009.

DANTAS, A. P. C. Tracoma: aspectos epidemiológicos no Brasil, 2009 - 2010 e perspectivas de controle. Dissertação Escola Nacional de Saúde Pública, Rio de Janeiro, RJ, 2013.

DEAN, D. et al. Predicting Phenotype and Emerging Strains among Chlamydia trachomatis Infections. Journal Emerging Infectious Diseases. Atlanta, V. 15, n. 9, September 2009

FERRAZ, L. C. B. et al. Tracoma em crianças do ensino fundamental no município de Bauru - Estado de São Paulo. Brasil. Arquivo Brasileiro de Oftalmologia, Bauru, v. 73, n. 5, Sept. - Oct. 2010.

GUYTON, A. C.; HALL, J. E. Tratado de Fisiologia Médica. 12 Ed. Rio de Janeiro, RJ: Elsevier, 2011.1151p.

HAFNER, L. M.; WILSON, D.P.; TIMMS, P. Development status and future prospects for a vaccine against Chlamydia trachomatis infection. Ed. Elsevier, 2013

JESUS, H. S. et al. Inquérito domiciliar de prevalência de tracoma em crianças do Distrito Federal, Brasil. Caderno de Saúde Coletiva, Rio de Janeiro, v. 21, n. 3, p. 318324, Sept. 2013.

KANSKI, J. J.; BROWLING, B. Oftalmologia Clínica. 6. Ed. Rio de Janeiro, RJ: Elsevier, 2008. 920p.

KOIZUMI, I. K. et al.. Prevalência do tracoma em pré-escolares e escolares no município de São Paulo. Revista de Saúde Pública. v. 39, n. 6, p. 937-942, 2005.

LAVETT, D. K. et al. Will the SAFE strategy be sufficient to eliminate trachoma by 2020 ? Puzzlements and possible solutions. The Scientific World Journal. article ID 648106 2013. 18p. 
LOPES, M. F. C. et al. Prevalência de tracoma entre escolares brasileiros. Revista de Saúde Pública. São Paulo, v. 47, n. 3, p. 451-459, Junho 2013.

LOPES, M. F. C. Tracoma: Situação epidemiológica no Brasil. Dissertação Instituto de Saúde Coletiva UFBA, Salvador, BA, 2008.

LUCENA, A. R.; CRUZ, A. A. V.; CAVALCANTI R. Estudo Epidemiológico do tracoma em comunidade da Chapada do Araripe - CE. Arquivo Brasileiro de Oftalmologia, v. 73, n. 3, 2010.

MACHADO, M. C. et al . A study of pentupmdemand in ophthalmology: Divinolândia Hospital/Unicamp. Revista brasileira de oftalmologia, Rio de Janeiro, v. 71, n. 6, Dec. 2012.

MACHARELLI, C. A. et al.. Spatial distribution of trachoma cases in the City of Bauru, State of São Paulo, Brasil, detected in 2006: defining Key areas for improvement of health resources. Revista da Sociedade Brasileira de Medicina Tropical. Uberaba, v. 46, n. 2, 2013.

MARIOTTI, S. P.; PASCOLINI, D.; ROSENUSSBAUNER, J. Trachoma: Global Magnitude of a Preventable Cause of Blindness. British Journal Ophthalmology. v. 93 , n. 5, p. 563-568, 2009.

MOLINA, D. L.; GUERRA, M. E. M.; LLORENTE, S. C. Importância clínica de Las Chlamydias. Revista Cubana de Medicina General Integral. La Habana, v. 29, n. 2, p. 214-225, abr./jun. 2013.

MILLMAN, K.L.; TAVARE, S. et al. Recombination in the ompA gene but not the omcB gene of Chlamydia contributes to serovar-specific differences in tissue tropism, imune surveillance, and persistence of the organism. J Bacteriol 183(20): 5997-6008. (2001).

MIRANDA, A.E.; GADELHA, A.M.J. et al. Impacto das infecções pela Chlamydia
PAULA, J. S.; MEDINA, N. H.; CRUZ, A. A. V. Trachoma among the Yanomami Indians. Brazilian Journal of Medical and Biological Research, Ribeirão Preto, v. 35, n. 10, Oct. 2002.

POLACK, S. et al. Mapping the global distribution of trachoma.Bulletin of the World Organization. v. 83, n. 1, p. 913-919, 2005.

PUTZ, C. Oftalmologia: Ciências Básicas. 2. Ed. Rio de Janeiro, RJ, Guanabara Koogan: Cultura Médica, 2011. 676p.

REILLY, L. A. et al., O. Preliminary evidence that synanthropic flies contribute to the transmission of trachoma - causing Chamydia trachomatis in Latin America. Caderno de Saúde Pública, [serial on the Internet]. , Rio de Janeiro, v. 23, n. 7, p. 16821688, 2007.

RIORDAN-EVA, P.; WHITCHER, J. P. Oftalmologia Geral de Vaughan \& Asbury. 17. Ed. Porto Alegre, RS: Artmed, 2011.463p.

SILVA, E. J. Ocorrência de Tracoma em escolares e o Impacto do treinamento de Habilidades Clínicas para Profissionais de Saúde em Turmalina, MG, Brasil. Dissertação. FCBS UFVJM, Diamantina, MG,2014.

SCHELlinI, S. A. et al. Prevalência e localização espacial dos casos de tracoma detectados em escolares de Botucatu, São Paulo - Brasil. Arquivo Brasileiro de Oftalmologia, São Paulo, v. 73, n. 4, 2010.

SCHELLINI, S. A.; SOUSA, R. L. F. Tracoma: ainda uma importante cauda de cegueira. Revista Brasileira de Oftalmologia. Rio de Janeiro, v. 71, n. 3, 2012.

THYLEFORS, B. et al. simple system for the assessment of trachoma and its complications. Bull World Organization. v. 65, n. 4, p. 477483, 1987.

WORLD HEALTH ORGANIZATION (WHO). Global Alliance for the Elimination WHO, Primary health care level management of on 
of Blinding Trachoma by 2020.Weekly Epidemiological Record, Geneva, v. 87, n. 17, p.161-168, 2012.

WORLD HEALTH ORGANIZATION (WHO). Planning for the global elimination of Trachoma (GET): report of a WHO Consultation Geneva; 1997.

YANOFF, M.; DUKER, J. Oftalmologia. 3 ed - Rio de Janeiro: Elsevier, 2011.1528p. 